\title{
A new chemical form of essential oil of Hyssopus officinalis L. (Lamiaceae) from Nigeria
}

\author{
Isiaka A. OGUNWANDE ${ }^{1 *}$, Guido FLAMINI ${ }^{2}$, Olubusayo O. ALESE $^{1}$, Pier L. CIONI ${ }^{2}$, \\ Akintayo L. OGUNDAJO ${ }^{1}$ and William N. SETZER ${ }^{3}$ \\ ${ }^{I}$ Natural Products Research Unit, Department of Chemistry, Faculty of Science, Lagos State University, \\ Badagry Expressway, P.M.B. 0001 Lasu Post Office, Ojo, Lagos, Nigeria. \\ ${ }^{2}$ Dipartimento di Scienze Farmaceutiche, sede Chimica Bioorganica e Biofarmacia, Via Bonanno 33, 56126 \\ Pisa, Italy. \\ ${ }^{3}$ Department of Chemistry, University of Alabama at Huntsville, Huntsville AL 35899, USA. \\ *Corresponding author; E-mail: isiaka.ogunwande@lasunigeria.org; Tel: + 2348059929304
}

\begin{abstract}
Essential oil obtained by hydrodistillation from the air-dried leaves of Hyssopus officinalis L. (Lamiaceae) collected in Ajangbadi area, West of Lagos, Nigeria, was analyzed comprehensively for its constituents by means of gas chromatography (GC) and gas chromatography-mass spectrometry (GC-MS). The monoterpene hydrocarbons, $\alpha$-pinene $(70.9 \%)$ and $\beta$-pinene $(10.9 \%)$ are the dominant constituents of the oil of $H$. officinalis. A cluster analysis was performed for comparison and characterization of $H$. officinalis essential oil from Nigeria with other oils reported in the literature from different locations across the world, and reveals chemical variation in this species with at least 8 different chemotypes. The compositional pattern of Nigerian oil sample was being reported for the first time and represents another chemotype of the oil of $H$. officinalis. (c) 2011 International Formulae Group. All rights reserved.
\end{abstract}

Keywords: GC-MS; $\alpha$-Pinene; $\beta$-Pinene; Chemotype

\section{INTRODUCTION}

Herb hyssop (Hyssopus officinalis L., family Lamiaceae) is a brightly coloured herbaceous plant of the genus Hyssopus native to Southern Europe, the Middle East, and the region surrounding the Caspian Sea. The plant is commonly used by beekeepers to produce rich and aromatic honey (Busari, 2006). Herb hyssop leaves are used as an aromatic condiment. The leaves have a lightly bitter taste due to its tannins, and an intense minty aroma. Due to its intensity, it is used moderately in cooking. The herb is also used to flavor liqueur. As a medicinal herb, hyssop has soothing, expectorant, and cough suppressant properties (Wyk and Wink, 2004). The $\alpha$-glucosidase inhibitory activity of this plant has been attributed to the presence of $(7 S, 8 S)$-syringoylglycerol-9-O-(6'-O-

cinnamoyl)- $\beta$ - D-glucopyranoside and $(7 S, 8 S)$ syringoylglycerol $\quad 9-O-\beta$-D-glucopyranoside (Matsuura et al., 2004).

The present work provides information on the chemical constituents of Hyssopus 
officinalis grown in Nigeria, and furthermore to compare and contrast the Nigerian $H$. officinalis sample with chemotypes previously reported from other parts of the world. This is part of our extensive research aimed at the characterization of the chemical constituents and biological activities of Nigerian medicinal plants and herbs as they are made available (Ogunbinu et al., 2010: Ogunwande et al, 2010).

\section{MATERIALS AND METHODS}

Plant materials

The leaves of $H$. officinalis were obtained from Adaluko area of Ajangbadi, Afromedia, Lagos, Nigeria, in March 2009. The plant sample was identified by curators at the Herbarium of the Department of Botany and Microbiology, University of Ibadan and the Herbarium Headquarters, Forestry Research Institute of Nigeria (FRIN), Ibadan, where voucher specimens have been deposited.

\section{Isolation of the volatile oils}

The air-dried plant sample was chopped and hydrodistilled for $4 \mathrm{~h}$ using a modified Clevenger-type apparatus. $700 \mathrm{~g}$ of the dried sample of the plant material were used for the hydrodistillation. The distilled oils were collected over water and stored in well capped bottles prior to analysis.

Gas chromatography (GC) and gas chromatograpgy-mass spectrometry (GCMS) analysis

The gas chromatography (GC) and the gas chromatography-mass spectrometry analysis follow the patterns previously described (Ogunbinu et al., 2010; Ogunwande et al., 2010).

\section{Identification of the constituents of the oil} Identification of the constituents was based on comparison of the retention times with those of authentic samples, comparing their linear indices relative to the series of $n$ hydrocarbons, and on computer matching against commercially available spectral (Adams, 2005). Further identifications were also made possible by the use of self constructed spectral library built up from pure substances and components of known oils and MS literature data (Masada, 1975; Jennings and Shibamoto, 1980; Davies, 1990). Moreover, the molecular weights of all the identified substances were confirmed by gas chromatography-chemical inonisation mass spectrometry, using methanol as CI ionizing gas.

\section{Numerical cluster analysis}

A cluster analysis was performed to determine the chemical relationships between the studied $H$. officinalis oil from Nigeria and the oils of this species reported in the literature from several other locations around the world. The $50 H$. officinalis samples were treated as operational taxonomic units (OTUs). The percentage composition of eleven main essential oil components (pinocamphone, isopinocamphone, pinocarvone, germacrene $\mathrm{D}$, limonene, methyl eugenol, 1,8-cineole, linalool, $\alpha$-pinene, $\beta$ pinene and bicyclogermacrene) were used to determine the chemical relationships between the different $H$. officinalis leaf oil samples by cluster analysis using the NTSYSpc software, version 2.2 (Rohlf, 2005). Correlation was selected as a measure of similarity, and the unweighed pair-group method with arithmetic average (UPGMA) was used for cluster definition. 
RESULTS

The hydrodistilation procedure afforded pale yellow oil in a yield of $0.24 \%$ (v/w). The percentage composition of the oil of $H$. officinalis is given in Table 1 . Constituents were listed in order of elution from HP-5 capillary column. Sixty-three compounds were identified, accounting for 99.3\% of total oil content. Monoterpenes $(89.4 \%)$ constituted the bulk of the oil, highly represented by $\alpha$ - and $\beta$-pinene $(70.9 \%$ and $10.9 \%$ respectively). Apart from limonene $(2.7 \%)$ and $\alpha$-campholenal (1.1\%), all the other monoterpene compounds occurred in amount less than $1 \%$. Sesquiterpene compounds were less common in the oil. $\beta$ Caryophyllene $(2.7 \%)$, caryophyllene oxide $(2.1 \%)$, viridiflorene $(1.1 \%), \quad \alpha$-guaiene $(1.0 \%)$ and guaiol $(1.0 \%)$ could be identified above $1 \%$. It should be noted that compounds such as pinocarvone, pinocamphone and isopinocamphone that are widely reported as dominant constituents of the oils from other parts of the world, occurred in the Nigerian sample in less significant quantitieis.

Table 1: Essential oil composition of Hyssopus officinalis.

\begin{tabular}{lll}
\hline Constituents & LRI $^{\mathbf{a}}$ & Perecentage (\%) \\
\hline isopentyl isovalerate & 876 & $\operatorname{tr}$ \\
$\alpha$-thujene & 931 & 0.6 \\
$\alpha$-pinene & 939 & 70.9 \\
camphene & 953 & 0.3 \\
thuja-2,4(10)-diene $*$ & 954 & 0.2 \\
benzaldehyde & 961 & $\operatorname{tr}$ \\
$\beta$-pinene & 980 & 10.9 \\
myrcene & 991 & 0.3 \\
$\alpha$-phellandrene & 1008 & $\operatorname{tr}$ \\
$\alpha$-terpinene & 1021 & $\operatorname{tr}$ \\
$p$-cymene & 1029 & 0.3 \\
limonene & 1034 & 2.7 \\
1,8 -cineole & 1037 & 0.2 \\
(Z)- $\beta$-ocimene & 1043 & $\operatorname{tr}$ \\
(E)- $\beta$-ocimene & 1054 & $\operatorname{tr}$ \\
$\alpha$-terpinene & 1064 & 0.1 \\
terpinolene & 1091 & 0.3 \\
linalool & 1103 & 0.2 \\
nonanal & 1106 & $\operatorname{tr}$ \\
$\beta$-thujone & 1114 & $\operatorname{tr}$ \\
exo-fenchol & 1117 & $\operatorname{tr}$ \\
$\alpha$-campholenal & 1131 & 1.1 \\
trans-pinocarveol & 1144 & $\operatorname{tr}$ \\
cis-verbenol & 1150 & $\operatorname{tr}$ \\
& &
\end{tabular}




\begin{tabular}{lll} 
trans-pinocamphone & 1160 & $\operatorname{tr}$ \\
pinocarvone & 1167 & 0.2 \\
borneol & 1170 & $\operatorname{tr}$ \\
cis-pinocamphone & 1178 & $\operatorname{tr}$ \\
4-terpineol & 1181 & $\operatorname{tr}$ \\
$p$-cymen-8-ol & 1188 & $\operatorname{tr}$ \\
$\alpha$-terpineol & 1193 & 0.4 \\
myrtenal & 1196 & 0.2 \\
verbenone & 1215 & $\operatorname{tr}$ \\
trans-carveol & 1223 & 0.2 \\
carvone & 1249 & $\operatorname{tr}$ \\
isobornyl acetate & 1290 & $\operatorname{tr}$ \\
trans-pinocarvyl acetate & 1302 & $\operatorname{tr}$ \\
$\alpha$-terpinyl acetate & 1355 & 0.3 \\
neryl acetate & 1370 & $\operatorname{tr}$ \\
$\alpha$-copaene & 1378 & 0.3 \\
geranyl acetate & 1388 & $\operatorname{tr}$ \\
$\beta$-elemene & 1394 & $\operatorname{tr}$ \\
$\alpha$-gurjunene & 1412 & $\operatorname{tr}$ \\
$\beta$-caryophyllene & 1421 & 2.7 \\
$\beta$-gurjunene & 1430 & $\operatorname{tr}$ \\
$\alpha$-guaiene & 1442 & 1.0 \\
$\alpha$-humulene & 1458 & 0.3 \\
$\gamma$-muurolene & 1478 & $\operatorname{tr}$ \\
germacrene D & 1483 & $\operatorname{tr}$ \\
$\beta$-selinene & 1486 & 0.2 \\
viridiflorene & 1493 & 1.1 \\
trans- $\gamma$-cadinene & 1515 & $\operatorname{tr}$ \\
$\delta$-cadinene & 1525 & 0.2 \\
spathulenol & 1578 & 0.5 \\
caryophyllene oxide & 1583 & 2.1 \\
guaiol & 1595 & 1.0 \\
humulene epoxide II & 1609 & $\operatorname{tr}$ \\
epi-10- $\gamma$-eudesmol & 1629 & $\operatorname{tr}$ \\
$\beta$-eudesmol & 1651 & $\operatorname{tr}$ \\
$\alpha$-eudesmol & 1655 & 0.2 \\
bulnesol & 1667 & 0.1 \\
hexadecanal & 1844 & $\operatorname{tr}$ \\
hexahydrofarnesylacetone & & 0.2 \\
\hline \multicolumn{1}{|c}{ aninear retention indices on HP-5 capillary column; } \\
$*$ Correct isomer not defined & &
\end{tabular}


Table 2: Percentage compositions of some Hyssopus officinalis oils.

\begin{tabular}{|c|c|}
\hline Principal Components & Authors \\
\hline $\begin{array}{l}\text { pinocamphone }(1.4-46.0 \%), \quad \text { isopinocamphone }(2.1-31.6 \%) \text {, } \\
\text { pinocarvone }(2.2-26.5 \%) \text {, germacrene D }(9.7-16.7 \%), \beta \text {-pinene } \\
(0.7-10.8 \%)\end{array}$ & Kerrola et al., $1994^{\text {a }}$ \\
\hline $\begin{array}{l}\beta \text {-pinene } \quad(4.07-8.85 \%), \quad \text { pinocamphone } \quad(5.56-31.23 \%), \\
\text { isopinocamphone }(39.21-55.17 \%) \text {, germacrene } \mathrm{D}(\mathrm{t}-9.02 \%)\end{array}$ & Jean et al., $1992^{\text {a }}$ \\
\hline $\begin{array}{l}\beta \text {-pinene } \quad(11.09-11.24 \%), \quad \beta \text {-phellandrene } \quad(7.88-12.67 \%), \\
\text { pinocamphone }(14.87-29.16 \%), \text { isopinocamphone }(13.20-28.54 \%)\end{array}$ & Venskutonis, $1995^{\mathrm{a}}$ \\
\hline pinocamphone $(18.1 \%)$, isopinocamphone $(17.4 \%)$ & Bourrel et al., $1995^{\text {a }}$ \\
\hline $\begin{array}{l}\text { pinocamphone }(50.5 \%) \text {, isopinocamphone }(17.9 \%), 1,8 \text {-cineole } \\
(3.2 \%)\end{array}$ & Bodrug et al., $1995^{\text {a }}$ \\
\hline isopinocamphone (5-> 50\%), pinocamphone (3->50\%), & Veres et al., $1997^{\mathrm{a}}$ \\
\hline $\begin{array}{l}\text { isopinocamphone (43.29\%), pinocamphone (16.79\%), } \beta \text {-pinene } \\
(16.31 \%)\end{array}$ & Glamoålija et al., 2005 \\
\hline $\begin{array}{l}\text { isopinocamphone (46.1\%), pinocamphone (15.3\%), germacren-D- } \\
11 \text {-ol }(6.1 \%) \text {, elemol }(5.2 \%)\end{array}$ & Cvijovic et al., 2010 \\
\hline $\begin{array}{l}\text { pinocamphone (14.1\%), isopinocamphone (44.7\%), germacren-D-4- } \\
\text { ol }(5.7 \%) \text { and elemol }(5.6 \%)\end{array}$ & $\begin{array}{ll}\text { Mitic and Dordevic, } \\
2001\end{array}$ \\
\hline $\begin{array}{lcccc}\alpha \text {-pinene } & (7.3 \%), \quad \beta \text {-pinene } & (5.3 \%), & \alpha \text {-terpinene } & (9.4 \%), \\
\text { pinocamphone }(46.7 \%), \text { isopinocamphone }(2.1 \%) & \end{array}$ & Sharma et al., $1963^{\text {a }}$ \\
\hline 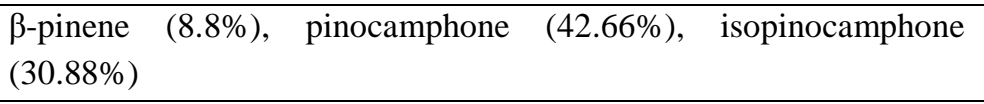 & Lawrence, $1995^{\text {a }}$ \\
\hline $\begin{array}{l}\text { Sample A: isopinocamphone }(53.12 \%), \quad \alpha \text {-terpineol }(7.4 \%) \text {, } \\
\text { pinocamphone }(4.7 \%) \\
\text { Sample B: isopinocamphone }(24.87 \%) \text {, pinocamphone }(14.41 \%) \text {, } \\
\text { elemol }(8.55 \%), \beta \text {-pinene }(7.81 \%)\end{array}$ & Nazari et al., 2008 \\
\hline $\begin{array}{l}\text { pinocamphone }(1.3-64.9 \%), \quad \text { isopinocamphone } \\
\text { pinocarvone }(0.1-16.9 \%), \beta-\text {-pinene }(2.8-13.2 \%)\end{array}$ & Chalchat et al., 2001 \\
\hline $\begin{array}{l}\text { pinocamphone }(4.4 \%), \text { isopinocamphone } \quad(43.3 \%), \\
(12.2 \%) \text { and } \beta \text {-pinene }(11.1 \%)\end{array}$ & Mazzanti et al., 1998 \\
\hline pinocamphone (49.1\%), $\beta$-pinene (18.4\%), isopinocamphone $(9.7 \%)$ & Garg et al., 1999 \\
\hline $\begin{array}{l}\text { 1,8-cineole ( } 48.2 \text { and } 39.6 \%) \text {, isopinocamphone (16.3 and } 28.0 \%), \\
\beta \text {-pinene (11.4 and 9.4\%) }\end{array}$ & Tsankova et al., $1993^{\mathrm{a}}$ \\
\hline $\begin{array}{l}\text { pinocamphone (34\% and } 18.5 \%) \text {, isopinocamphone }(3.2 \% \text { and } 29 \%) \\
\text { and } \beta \text {-pinene }(10.5 \% \text { and } 10.8 \%) \text {, linalool }(0.2 \% \text { and } 7.9 \%) \text { and } \\
\text { camphor }(0.3 \% \text { and } 5.3 \%)\end{array}$ & Fraternale et al., 2004 \\
\hline $\begin{array}{l}\text { pinocarvone }(36.3 \%) \text {, pinocamphone }(19.6 \%), \beta \text {-pinene }(10.6 \%) \text {, } \\
1,8 \text {-cineole }(7.2 \%) \text { and isopinocamphone }(5.3 \%)\end{array}$ & Ozer et al., 2005 \\
\hline pinocamphone $(1.72 \%)$, isopinocamphone $(43.26 \%)$, limonene & Salvatore et al., $1997^{\text {a }}$ \\
\hline
\end{tabular}




\begin{tabular}{|c|c|}
\hline \multicolumn{2}{|l|}{$(12.18 \%)$, methyl eugenol (4.01\%) } \\
\hline pinocamphone, camphor and $\beta$-pinene & Schultz and \\
\hline & Biskup, 1991 \\
\hline$\beta$-pinene $(9.60 \%)$, limonene $(37.40 \%)$, methyl eugenol $(38.30 \%)$ & Gorunovic et al., $1995^{\mathrm{a}}$ \\
\hline$\beta$-pinene $(16.82 \%), 1,8$-cineole $(52.89 \%)$, myrcenol $(3.1 \%)$ & Garcia et al., $1995^{\mathrm{a}}$ \\
\hline $\begin{array}{l}\text { 1,8-cineole with low amount of pinocamphone, isopinocamphone } \\
\text { and pinocarvone }\end{array}$ & Lopez et al., 2008 \\
\hline$\beta$-pinene and limonene $(1->60 \%)$ & Veres et al., $1997^{\mathrm{a}}$ \\
\hline linalool $(51.7 \%), 1,8$-cineole $(12.3 \%)$ and limonene $(5.1 \%)$ & Mazzanti et al., 1998 \\
\hline $\begin{array}{l}\text { linalool }(49.00-51.65 \%), 1,8 \text {-cineole }(11.92-14.91 \%), \text { limonene } \\
(4.99-6.02 \%)\end{array}$ & Salvatore et al., $1997^{\mathrm{a}}$ \\
\hline $\begin{array}{l}\alpha \text {-pinene }(70.9 \%) \text { and } \beta \text {-pinene }(10.9 \%), \text { limonene }(2.7 \%) \text {, } \\
\text { pinocamphone and isopinocamphone }(\operatorname{tr}), \text { pinocarvone }(0.2 \%)\end{array}$ & Present Study \\
\hline
\end{tabular}

* Quantitative data not available, ${ }^{\text {a }}$ Cited in B.M. Lawrence, 2003

Table 3: Chemotypes of Hyssopus officinalis oils.

\begin{tabular}{|c|c|c|}
\hline Chemotypes & Forms & \multirow{7}{*}{$\begin{array}{l}\text { References } \\
\text { Kerrola et al., } 1994^{\mathrm{a}}\end{array}$} \\
\hline \multirow{7}{*}{$\begin{array}{l}\text { Oils with } \\
\text { abundance of } \\
\text { pinocarvone }\end{array}$} & pinocarvone $>$ isopinocamphone & \\
\hline & germacrene $\mathrm{D}>\beta$-pinene & \\
\hline & pinocarvone $>$ germacrene $\mathrm{D}$ & \\
\hline & isopinocamphone $>\beta$-pinene & \\
\hline & pinocarvone $>$ isopinocamphone & \\
\hline & germacrene D > pinocamphone & \\
\hline & $\begin{array}{l}\text { pinocarvone }>\text { pinocamphone }>\beta \text {-pinene }> \\
1,8 \text {-cineole }\end{array}$ & Ozer et al., 2005 \\
\hline \multirow{7}{*}{$\begin{array}{l}\text { Oils which are } \\
\text { rich in } \\
\text { pinocamphone }\end{array}$} & $\begin{array}{l}\text { pinocamphone }>\text { isopinocamphone }>\beta \text { - } \\
\text { pinene }>\beta \text {-phellandrene }\end{array}$ & $\begin{array}{l}\text { Venskutonis, } 1995^{\mathrm{a}} \text {, Lawrence, } \\
\text { 1995, Chalchat et al., 2001, } \\
\text { Bourrel et al., } 1995^{\mathrm{a}}\end{array}$ \\
\hline & $\begin{array}{l}\text { pinocamphone }>\text { germacrene } \mathrm{D}> \\
\text { pinocarvone }\end{array}$ & Kerrola et al., $1994^{\mathrm{a}}$ \\
\hline & pinocamphone $>$ germacrene $\mathrm{D}>\beta$-pinene & Kerrola et al., $1994^{\mathrm{a}}$ \\
\hline & $\begin{array}{l}\text { pinocamphone }>\quad \beta \text {-pinene }> \\
\text { isopinocamphone }\end{array}$ & $\begin{array}{l}\text { Fraternale et al., 2004, Garg et } \\
\text { al., } 1999\end{array}$ \\
\hline & $\begin{array}{l}\text { pinocamphone }>\alpha \text {-terpinene }>\alpha \text {-pinene }> \\
\beta \text {-pinene }\end{array}$ & Sharma et al., $1963^{\mathrm{a}}$ \\
\hline & $\begin{array}{l}\text { pinocamphone }>\text { isopinocamphone }>1,8 \text { - } \\
\text { cineole }\end{array}$ & Bodrug et al., $1995^{\mathrm{a}}$ \\
\hline & pinocamphone $>$ camphor $>\beta$-pinene & $\begin{array}{l}\text { Schultz and Stahl-Biskup, } \\
1991\end{array}$ \\
\hline
\end{tabular}




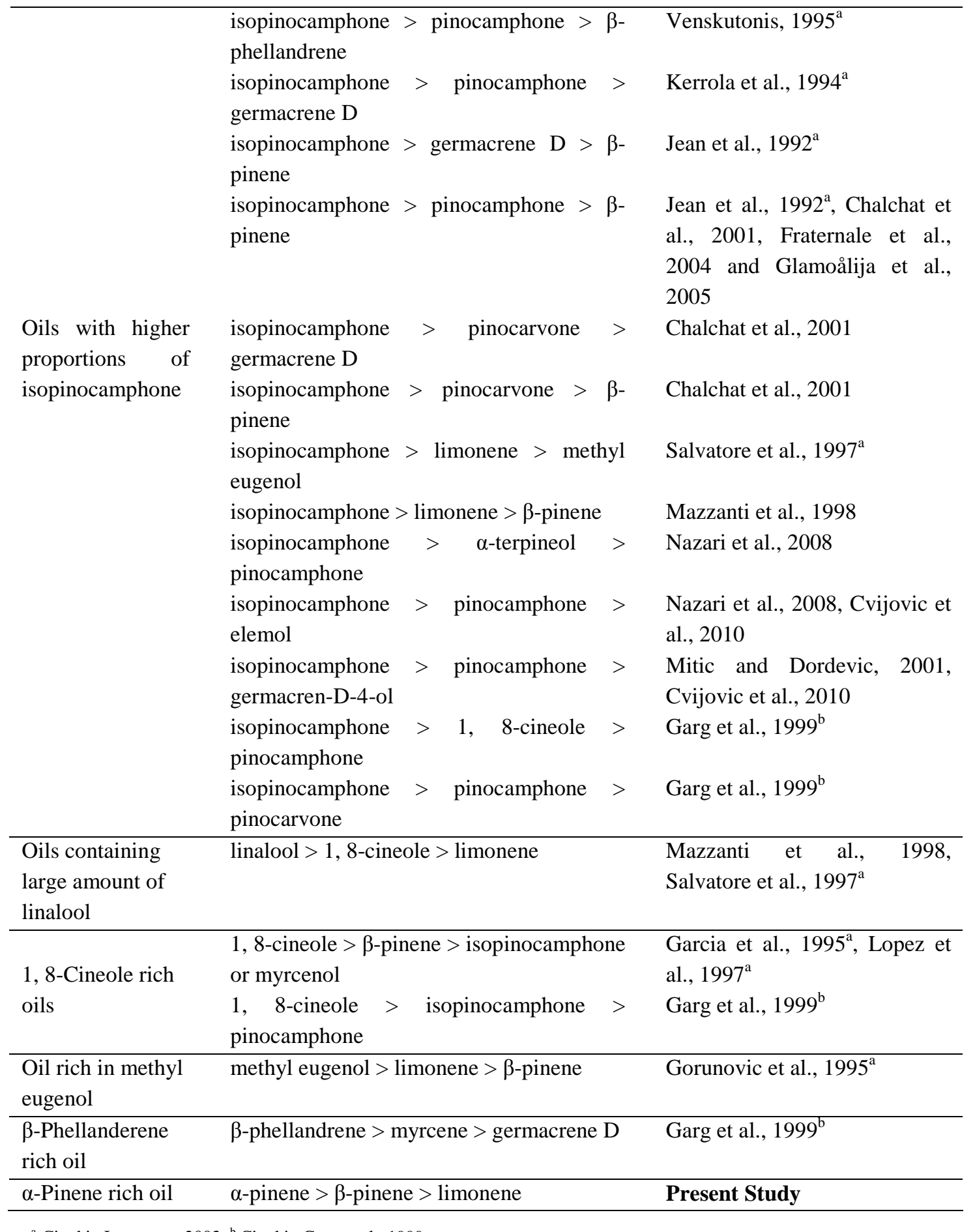

${ }^{\mathrm{a}}$ Cited in Lawrence, 2003, ${ }^{\mathrm{b}}$ Cited in Garg et al., 1999 


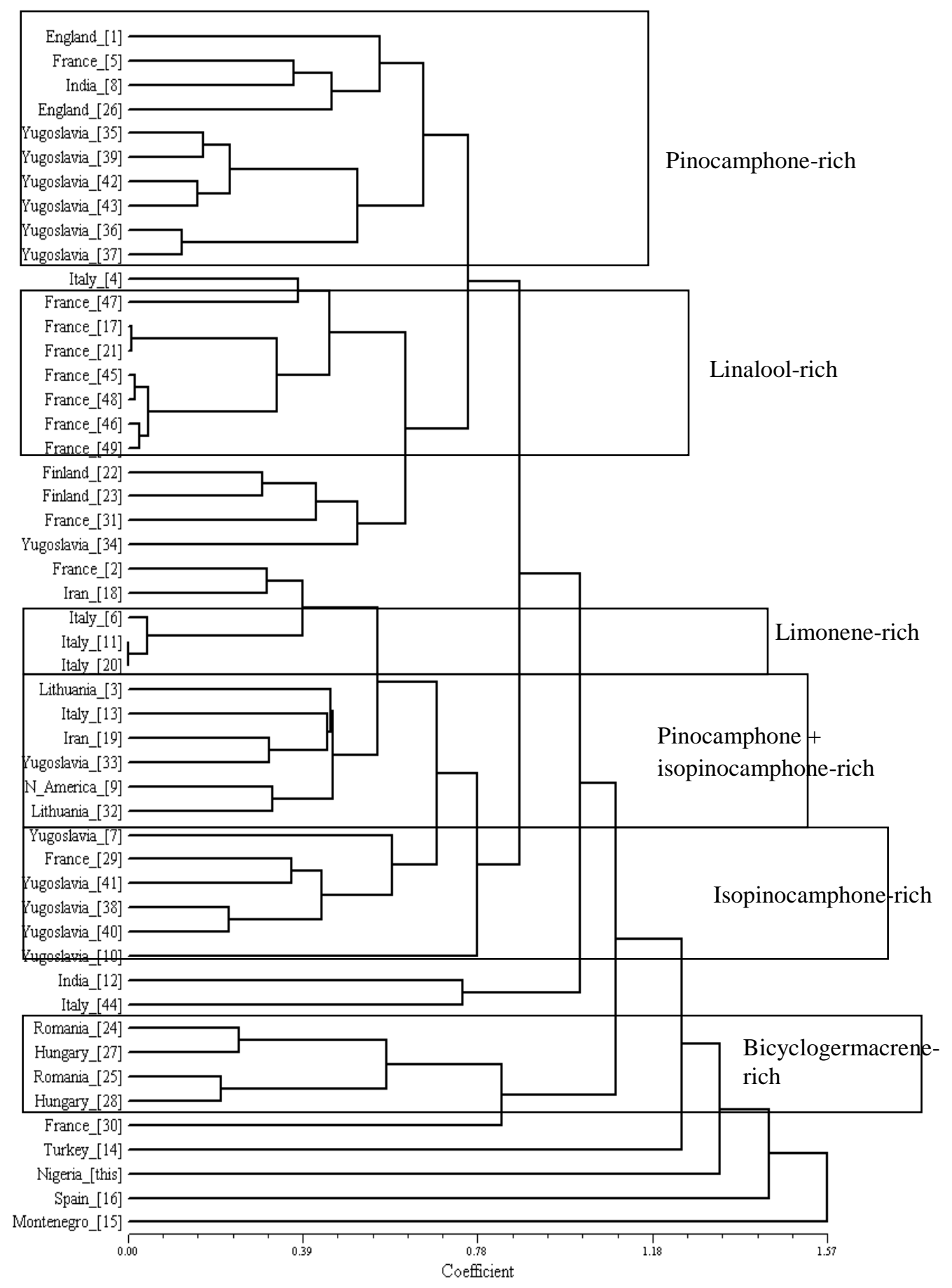

Fig 1: Cluster analysis of the oils of Hyssopus officinalis. 


\section{DISCUSSION}

The abundance of monoterpenoid compounds in the present oil sample is in agreement with previous literature reports. Previous authors (Schulz and Stahl-Biskup, 1991; Mazzanti et al., 1998; Garg et al., 1999; Chalchat et al., 2001; Mitic and Dordevic, 2002; Lawrence, 2003; Fraternale et al., 2004; Glamoålija et al., 2005; Ozer et al., 2005; Lopez et al., 2007; Nazari et al., 2008; Cvijiovic et al., 2010) have shown that the oil composition of $H$. officinalis showed large variations in the relative concentration of its major components that can be related to genotype, location and climatic conditions, although the presence of the bicyclic monoterpene ketones, pinocamphone and isopinocamphone remains peculiar (Table 2). $\alpha$-Pinene as occurred in the present oil sample has not been described to be the major constituent of the oils of $H$. officinalis and its varieties. It is evident from the literature reports mentioned above and the present study that several chemotypic forms of the oils are discernible (Table 3 ). In the present oil sample from Nigeria, except for its $\beta$-pinene content, the major constituents of the oils of Hyssopus species, such as pinocamphone, isopiocamphone, 1, 8-cineole, pinocarvone, linalool and limonene, as reported by earlier researchers were either found in insignificant quantities or completely absent.

The cluster analyses of the principal components are depicted in Fig 1. There are some apparent clusters: pinocamphone-rich $(1,5,8,26,35,36,37,39,42,43)$, linaloolrich $(17,21,45,46,47,48,49)$, limonene-rich $(6,11,20)$, pinocamphone + isopinocamphone $(3,9,13,19,32,33)$, isopinocamphone-rich (7, $10,29,38,40,41)$, and bicyclogermacrenerich $(24,25,27,28)$.

\section{Conclusion}

It could be seen that the compositional pattern of the present oil sample does not fit into any of the clusters and is unique. This may represent another chemotype of the essential oil of $H$. officinalis.

\section{REFERENCES}

Adams RP. 2001. Identification of Essential Oil Components by Gas Chromatography/ Mass Spectrometry $\left(4^{\text {th }}\right.$ edn). Allured Publishing Corporation: Carol Stream, Illinois.

Busari AO. 2006. Compendium of Southwest Nigerian Medicinal Plants. Al-Faruq Publisher: Ibadan, Nigeria.

Chalchat J-C, Adamovic D, Gorunovic MS. 2001. Composition of oils of three cultivated forms of Hyssopus officinalis endemic in Yugoslavia: $f$. albus Alef., $f$. cyaneus Alef. and f. ruber Mill. J. Essent. Oil Res, 13(6): 419-421.

Cvijovic M, Djukic D, Mandic L, AcamovicDjokovic G, Pesakovic M. 2010. Composition and antimicrobial activity of essential oils of some medicinal and spice plants. Chem. Nat. Compd., 46(3): 481483.

Davies NW. 1990. Gas chromatographic retention indices of monoterpenes and sesquiterpenes on Methyl Silicone and Carbowax 20M phases. J. Chromatogr. A., 503: 1-24.

Fraternale D, Ricci D, Epifano F, Curini M. 2004. Composition and antifungal activity of two essential oils of hyssop (Hyssopus officinalis L.). J. Essent. Oil Res., 16(6): 617-622.

Garg SN, Naqvi AA, Singh A, Ram G, Kumar S. 1999. Composition of essential oil from an annual crop of Hyssopus officinalis grown in Indian plains. Flav. Fragr. J., 14(3): 170-172.

Glamoalija JM, Sokovia MD, Vukojevia JB, Milenkovia IM, Brkia DD, van Griensven LJD. 2005. Antifungal activity of essential oil of Hyssopus officinalis L. against Mycopathogen Mycogone perniciosa (Mang). Proc. Nat. Sci, Matica Srpska Novi Sad., 109(2): 123-128. 
Jennings W, Shibamoto T. 1980. Qualitative Analysis of Flavour Volatiles by Gas Capillary Chromatography. Academic Press: New York.

Lawrence BM. 2003. Essential Oils 19952000. Allured Publishing Corporation: Carol Stream, Illinois.

Lopez N, Cerpa M, Cocero MJ, Marr, R, Gamse T. 2007. Extraction of Spanish Hyssop with Supercritical $\mathrm{CO}_{2}$. In Proceedings of $38^{\text {th }}$ International Symposium on Essential Oils, Graz, Austria; 49.

Massada Y. 1975. Analysis of Essential Oils by Gas Chromatography and Mass Spectrometry. John Wiley \& Sons: New York.

Matsuura H, Miyazaki H, Asakawa C, Amano M, Oshihara TY, Mizutani J. 2004. Isolation of $\alpha$-glucosidase inhibitors from hyssop (Hyssopus officinalis). Phytochem., 65(1): 91-97.

Mazzanti G, Battinelli L, Salvatore G. 1998. Antimicrobial properties of the linaloolrich essential oil of Hyssopus officinalis L. var decumbens (Lamiaceae). Flav. Fragr. J., 13(5): 289-294.

Mitic V, Dordevic S. 2002. Essential oil composition of Hyssopus officinalis L. cultivated in Serbia. Facta Universitatis, Series Phy. Chem. Technol., 2(2): 105108.

Nazari F, Shaabani Sh, Kazemizadeh Z, Jafari E, Alnajjar Z. 2008. Comparison of chemical composition from Hyssopus officinalis L. cultivated in different locations of Iran. In Proceedings of $39^{\text {th }}$
International Symposium on Essential Oils, Quedlinburg, Germany; 106.

Ogunbinu AO, Okeniyi SO, Flamini G, Cioni PL, Ogunwande IA. 2010. Monoterpenoid Constituents of the volatile oils of Cynometra megalophylla Harms., Caesalpinia pulcherrima L. Swartz and Pachylobus edulis G. Don., growing in Nigeria. J. Essent Oil Res., 22(6): 536539.

Ogunwande IA, Flamini G, Cioni PL, Omikorede O, Azeez RA, Ayodele AA, Yusuff KO. 2010. Aromatic Plants from Nigeria: Constituents of Cassia alata (Linn.) Roxb. and Helianthus annuus L. Rec. Nat. Prod., 4(4): 211-217.

Ozer H, Sahin F, Hamdullah KLC, Gulluce M. 2005. Essential oil composition of Hyssopus officinalis L. subsp. angustifolius (Bieb.) Arcangeli. Flav. Fragr. J., 20(1): 42-44.

Rohlf JF. 2005. NTSYSpc, Numerical Taxonomy and Multivariate Analysis System. Applied Biostatistics Inc: New York.

Schulz G, Stahl-Biskup E. 1991. Essential oils and glycosidic bound volatiles from leaves, stems, flowers and roots of Hyssopus officinalis L. (Lamiaceae). Flav. Fragr. J., 6(1): 69-73.

Wyk VB-E, Wink M. 2004. Medicinal Plants of the World. Timber Press Inc; 177. 